\title{
Analytical Electron Microscopy: Rivaling / Complementing Atom Probe Tomography
}

\author{
MG Burke ${ }^{1}$ and Joven J H Lim² \\ 1. Materials Performance Centre, University of Manchester, Oxford Road, Manchester, UK. \\ 2. UK Atomic Energy Authority, Culham Science Centre, Abingdon, UK
}

With the development of EMMA by Duncumb [1], followed by EMMA-4 [2], and quantification of energy dispersive X-ray (EDX) spectra in the TEM, the field of analytical electron microscopy was born. The ability to perform conventional TEM and electron diffraction analyses was complemented by the incorporation of STEM detectors, secondary electron and backscattered electron detectors, and an energy dispersive X-ray spectrometer to provide multi-modal microstructural and microchemical $(Z>11)$ characterisation of materials. Numerous excellent texts accompanied the rapid developments in AEM, such as Williams [3]. For nearly 45 years, AEM techniques have been applied to a very broad range of metals, alloys, minerals and other materials (including biomaterials), and has been essential in the development and optimization of materials and devices.

The growth of AEM has continued over the past several decades, particularly with the development of Si Drift Detectors (SDDs), new detector geometries, advances in FEG technology and in microscope design. Thus, improvements in elemental detectability, spatial resolution, specimen preparation has made the ability to detected nanoscale segregation "routine" in materials characterisation and analysis. Previously, atom probe field-ion microscopy (AP-FIM) provided the ultimate high spatial resolution quantitative microanalysis data, albeit complicated by the very limited volumes analyzed as the data analysis. But the introduction of atom probe tomography (APT) [4-6] revolutionized atom probe analysis by enabling the 3D reconstruction of the field-evaporated ions via the use of position-sensitive ion detectors. Thus, APT was the ultimate technique for nanoscale analysis, with vast improvements in data acquisition and volume of analysis compared to the AP-FIM technique.

Advance AEMs now provide the ability to perform STEM-EDX spectrum imaging analyses at high spatial resolution over volumes that are far greater than APT analysis volume while maintaining elemental sensitivity $(Z>5)$. Thus, the detection of nanoscale segregation to defects and interfaces is now routinely possible. Similarly, complex precipitate compositions that could only be measured via APFIM or APT can now be analysed via high spatial resolution STEM-EDX nanoscale analysis, with excellent agreement to atom probe analyses. The remarkable capabilities of advanced AEMs for the characterisation of nanoscale precipitates in complex Ni-base superalloys (such as Alloy 718) and the ability to detect nanoscale segregation to dislocations, cavities and irradiation-induced defects in proton and ion-irradiated alloys will be discussed. Figure 1 provides an example from the FEI Talos F200 with Super X of elemental maps extracted from a STEM-EDX spectrum image dataset for fine $\gamma^{\prime}$ and $\gamma$ ' precipitates in Alloy 718. These STEM-EDX data revealed that the $\gamma$ " precipitates (nominally $\mathrm{Ni}_{3}(\mathrm{NbTi})$ ) also contained $\mathrm{Al}$, and the $\gamma$ ' precipitates (nominally $\mathrm{Ni}_{3}(\mathrm{AlTi})$ ) contained $\mathrm{Nb}$, in excellent agreement with published AP data [7]. An example of proton-irradiation-induced Ni and Si segregation to dislocation loops and cavities is provided in Figure 2. These examples demonstrate the powerful nature of advanced AEM (STEM-EDX microanalysis) as it now can provide direct evidence for nanoscale features and segregation whereas previously such data were only obtainable via atom probe analysis [8]. 
References:

[1] P Duncumb, Proc. $5^{\text {th }}$ Int. Congr. on EM (Academic Press, NY) (1962) KK4.

[2] G Cliff and GW Lorimer, Proc. $5^{\text {th }}$ Eur. Congr. On EM - Manchester (1972) p. 140.

[3] DB Williams, Practical Analytical Electron Microscopy in Materials Science (Philips Electron Optics Publishing Group, Mahwah).

[4] A Cerezo, TJ Godfrey and GDW Smith, J. Phys. 49 (C6) (1988), p. 25.

[5] D Blavette et al., Rev. Sci. Inst. 64 (1993), p. 2911.

[6] TF Kelly et al., Microsc. Microanal. 10 (2004), p. 373.

[7] MG Burke and MK Miller, Superalloys 718, 625 \& Various Derivatives, (TMS, 1991), p. 337.

[8] The authors acknowledge the support from EPSRC Grant No. EP/N017854/1 and EP/N017854/1.

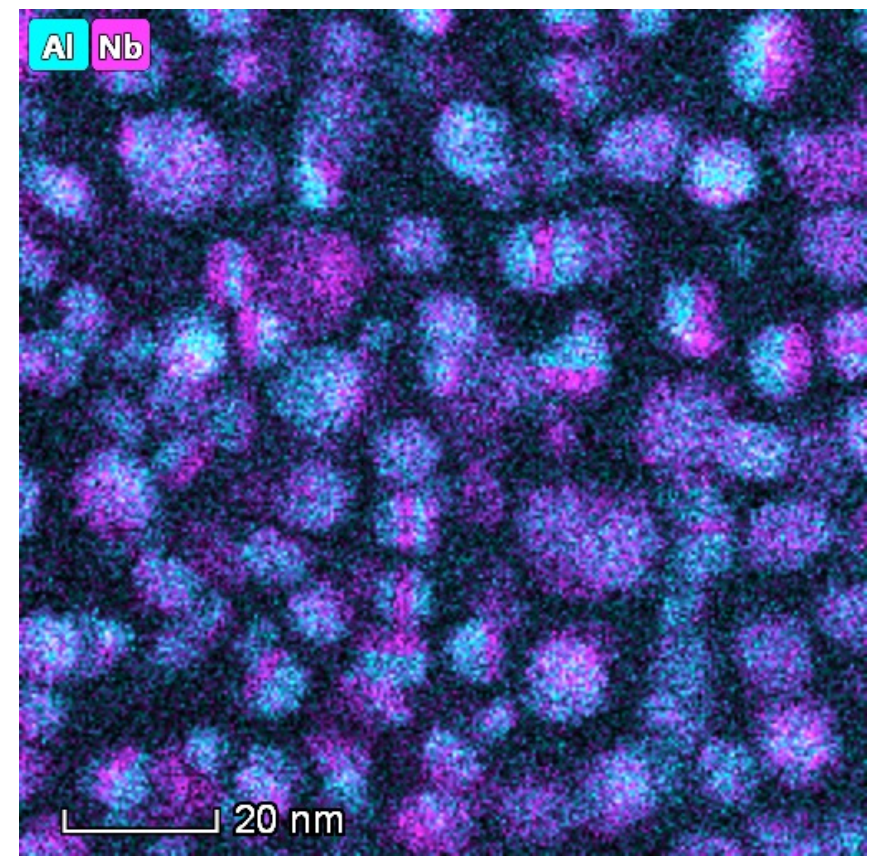

Figure 1. STEM-EDX elemental maps for $\mathrm{Nb}$ and Al obtained from Alloy 718 on the Titan G2 $80 / 200$ FEG-AEM. The highest Al enrichment is associated with $\gamma^{\prime}$.

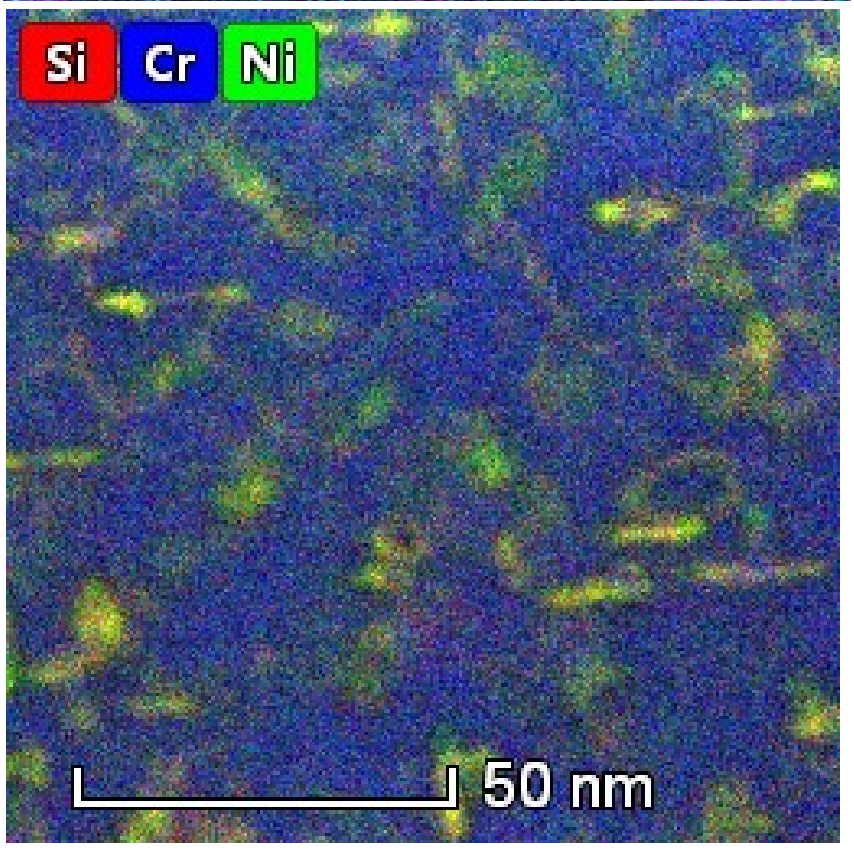

Figure 2. STEM-EDX elemental maps for $\mathrm{Si}, \mathrm{Cr}$. and $\mathrm{Ni}$. Note the decoration of dislocation loops (edge - on) and isolated dislocation with $\mathrm{Si}$ and $\mathrm{Ni}$. 